\title{
Téoros
}

Revue de recherche en tourisme

\section{Qui sont-ils ? Que font-ils ?}

\section{Sylvie Houle, Marc-André Lalande et Hélène Terroux}

Volume 1, numéro 2, 2e trimestre 1982

La recherche en tourisme : forces et faiblesses

URI : https://id.erudit.org/iderudit/1080855ar

DOI : https://doi.org/10.7202/1080855ar

Aller au sommaire du numéro

Éditeur(s)

Université du Québec à Montréal

ISSN

0712-8657 (imprimé)

1923-2705 (numérique)

Découvrir la revue

Citer ce document

Houle, S., Lalande, M.-A. \& Terroux, H. (1982). Qui sont-ils ? Que font-ils ?

Téoros, 1(2), 13-19. https://doi.org/10.7202/1080855ar d'utilisation que vous pouvez consulter en ligne.

https://apropos.erudit.org/fr/usagers/politique-dutilisation/ 


\title{
Qui sont-ils? Que font-ils?
}

\author{
Par Sylvie Houle, Marc-André Lalande et Hélène Terroux
}

\section{Présentation sommaire et sélective de quelques recherches intéressantes et des principaux lieux où se réalisent des recherches et des études dans le domaine du tourisme.}

Les articles précédents ont mis en évidence les problèmes que rencontre la recherche en tourisme au Québec. Malgré cela, on dénombre ces dernières années plusieurs études et quelques recherches (généralement des recherches appliquées, parfois des recherches fondamentales) sur divers aspects du phénomène touristique au Québec et au Canada. Elles furent réalisées par des firmes de consultants pour le compte de divers ministères et des entreprises privées, ou directement par les services de recherche des ministères concernés. Le secteur associatif et le milieu universitaire ne sont pas en reste sur ce plan et ont contribué aussi à l'avancement des connaissances sur le tourisme.

Les pages qui vont suivre donnent un aperçu des "producteurs" de recherches touristiques et de leur "récolte". Le focus est mis à l"occasion sur une recherche qui, pour les auteurs, semble digne d'intérêt.

II ne faut pas trouver dans ces pages un inventaire exhaustif de la recherche en tourisme mais bien une présentation sommaire et sélective.
1) Les firmes de consultants

Voici une liste partielle de firmes québecoises et canadiennes qui ont effectué récemment des études dans le domaine touristique. On mentionne, pour chacune une réalisation et l"organisme qui l'a commandée.

\section{Market Facts of Canada Ltd \\ 1240, Bay Street \\ 7 e étage \\ Toronto, Ontario}

Etude sur les possibilités du marché américain.

Commandée par l'Office de Tourisme du Canada. 1982

Pluram

81, rue St-Pierre

C.P. 158 , Succ. B

Québec. Québec

G1K 7A6

Etude sur le transport de rlle aux Coudres.

Commandée par le ministére des Transports du Québec. 1980

\section{Sodem}

1380, rue Gilford

Montréal, Québec

H2J $1 R 7$

Etude du Mont Sir Wilfrid Pelletier au Quebec, sur le potentiel et le déplacement des visiteurs.

Commandée par le ministère de I'Industrie, Commerce et Tourisme. 1981

\section{Sorecom}

84 ouest $_{\text {r }}$ rue Notre-Dame

Suite 500

Montréal, Québec

$\mathrm{H} 2 \mathrm{Y} 1 \mathrm{~S} 6$

Etude aupres des installations, (il s'agit d'une étude de clientèle en rapport avec les visites des installations hydroélectriques)

Commandée par l'Hydro-Québec, 1980
Sores

1. Complexe Desjardins

C.P. 10 , Succ. Desjardins

Montréal, Québec

H5B 1 C8

Elaboration d'un plan marketing pour un centre hôtelier et récréatif à Morin Height.

Commandée par le Développement de Morin Height. 1980

Sotar

3333, rue du Souvenir

Chomedey, Québec

H7V $1 \times 1$

Station touristique du Mont-Tremblant. plan de développement len collaboration avec SECOR).

Etude commandée par la Station

Touristique du Mont-Tremblant. 1981

Thorne Stevenson and Kellog

485, rue Albert

Suite 1000 ,

Ottawa, Ontario

Etude du marche des congres et réunions d'affaires au Canada.

Étude commandée par l'Office de Tourisme du Canada, (étude en cours)

Travel Data of Toronto Ltd.

188 est. Eglinton ave

Suite 501

Toronto. Ontario

M4P 2X7

Voyages d'agrément des Canadiens.

Étude commandée par l'Office de Tourisme du Canada. 1981 


\section{2) Les services gouvernementaux de recherche}

Par l'entremise du Bulletin des recherches touristiques publié par I'Office de Tourisme du Canada (OTC)". il est possible de sinformer sur les recherches en tourisme menées au Canada par les divers services gouvernementaux. L'énumération cidessous comprend quelques recherches parmi les plus récentes:

\begin{abstract}
Alberta
Etude de la campagne publicitaire générale sur le tourisme de 1981.

Le ministère du Tourisme de l'Alberta a commandité en février et septembre 1981 une série d'annonces publicitaires invitant le lecteur à retourner un coupon s'il désirait recevoir gratuitement une pochette d'information sur l'alberta. L'étude a servi à évaluer dans quelle mesure les annonces ont réussi à susciter une demande pour ces pochettes et à amener en conséquence les personnes intéressées à concrétiser leur voyage en Alberta.
\end{abstract}

Ile-du-Prince-Edouard

Enquête sur les sorties touristiques 1981

Une enquête sur le sujet a été effectuée du 15 mai au 31 octobre 81 . Les données démographiques et les données concernant les habitudes de voyages et les dépenses effectuées ont été recueillies aux trois points de sortie de l'île, soit à l'aéroport de Charlottetown et aux ports de traversiers de Borden et Wood Islands.

\section{Manitoba}

Apercu du trafic touristique entrant au Manitoba par la route numéro 1 est et ouest, par mois, en 1980 et 1981 .

En 1980 et 1981, la direction de la Recherche et de l'Analyse économique du ministère du Développement économique et du Tourisme a fait le relevé du trafic touristique entrant dans la province du Manitoba par la route numéro 1 est (à la frontière du Manitoba et de l'Ontario) et par la route numéro 1 ouest là la frontière du Manitoba et de la Saskatchewan), avec pour objectif d'établir une base de données portant sur douze mois pour les enquêtes futures.
Nouvelle-Écosse

Enquête sur le Salon du tourisme de plein-air

L'enquête tente d'établir le nombre de personnes qui sont venues ou pensent venir en Nouvelle-Écosse à I'occasion du Salon du tourisme de plein-air, et d'évaluer la position qu'occupe la Nouvelle-Écosse par rapport au Nouveau-Brunswick, au Québec et à I'Ontario en tant que destination touristique de plein-air.

\section{Québec}

Sondage sur la perception du produit touristique québécois qu'ont les Américains ayant fait un voyage d'agrément au cours des 24 derniers mois et sur le niveau de pénétration de la campagne publicitaire U.S. pour l'été 1981.

Ce sondage a été réalisé auprès des Américains des agglomérations de New-York, Chicago, Hartford, Providence et Washington. II vise à avoir des précisions sur le comportement et les attentes des Américains lors de voyages d'agrément et de mieux connaître leurs opinions sur le produit touristique québécois.

\title{
Étude sur le réseau d'accueil et d'information touristique au Québec
}

\author{
Cette étude fut réalisée par la firme Drouin. Paquin et associés Ltée pour le \\ compte du ministère de l'Industrie, Commerce et Tourisme, en 1980.
}

Le mandat des consultants consistait à faire l'étude du réseau des centres d'accueil et d'information touristiques en vue de formuler des recommandations pour son amélioration. Le présent résumé ne traite que de la première partie de l'étude.

Les consultants examinèrent premièrement les deux formes de réseaux de centres existants. D'abord. il y a le réseau gouvernemental dont les établissements sont à vocations nationale et frontalière. Les déficiences que soulignent les consultants sont les suivantes: absence de planification. installations inadéquates en fonction des besoins, localisation problématique, signalisation déficiente, sousutilisation des ressources humaines. mauvaise concertation des intervenants. politique linguistique contraignante, outils de travail incomplets, dépendance trop grande des services de la fonction publique. $\mathrm{A}$ celà, ilss ajoutent un manque de souplesse aux nouvelles conditions du marché.
Le second réseau qui opère avec un permis de la direction générale du Tourisme (MICT), est constitué d'environ 150 établissements saisonniers et permanents. Aux déficiences physiques déjà relevées pour le réseau précédent, les consultants ajoutent des difficultés majeures d'organisation (centres à mission non touristique, responsabilités non déterminées entre les centres, moyens inégaux et insuffisants), des difficultés de collaboration (avec les centres d'accueil des parcs et des sites historiques fédéraux et entre la direction générale du Tourisme, et le service des Parcs), et finalement des problèmes politiques (politique floue du ministère au sujet de l'opération de ces centres).
Les consultants tracent ensuite le rôle que jouent actuellement les Associations touristiques régionales. II est mentionné que de bonnes initiatives ont été prises dans les régions comme, par exemple, de monter des centrales téléphoniques d'informations et d'évaluer la qualité des réseaux locaux.

Finalement, il est constaté que compte tenu de leurs moyens, les ATR semblent efficaces mais qu'elles éprouvent certains handicaps: mauvaises relations avec le gouvernement, découpage incorrect des régions, ... Les consultants terminent ensuite cette première partie par un examen critique des solutions que proposent les ATR, avant d'aborder dans la deuxième partie leurs propres solutions aux problèmes.

Deux ans plus tard, quelles conclusions devons-nous tirer de cet éloquent rapport qui ne fit que trop peu parler de lui?

(résumé de Daniel Bibeau) 


\section{Saskatchewan}

Etude sur les utilisateurs des lieux historiques de la Saskatchewan et dénombrement des véhicules récreatifs.

II s'agit d'un rapport d'étude sur les utilisateurs d'un lieu historique de la Saskatchewan et sur le dénombrement des véhicules récréatifs sur les autoroutes, no 11 et no 2. Le lieu historique de la Saskatchewan est un territoire d'environ $130 \mathrm{~km}^{2}\left(50 \mathrm{mi}^{2}\right)$ où se trouvent de nombreux sites historiques (notamment les sites qui ne réfèrent à la deuxième rébellion de Riel).

\section{Terre-Neuve}

La division de la planification et de I"évaluation du ministère du Développement, à l'été 1981, fera deux enquêtes, dont l'une portera sur le nombre des sorties en automobile et l'autre sur le nombres de sorties en avion, en 1982. Les résultats seront publiés au début de 1983.

\section{2) Le milieu associatif}

Les associations sans but lucratif oeuvrant dans le domaine du tourisme réalisent plusieurs études et recherches. L'article de Nicole Chenette et de ses collaborateurs, dans le présent numéro, révèle l'intérêt manifeste des associations pour la recherche, qu'il s"agisse des associations touristiques regionales, des chambres de commerce ou des associations concernées par le phénomène du tourisme social (pour ne nommer que celles-lâ).
La plupart des associations québécoises de tourisme social sont membres du secteur "tourisme" du Regroupement des organismes nationaux de loisir du Québec. Le secteur "tourisme" est une table de concertation qui contribue au développement des associations et du tourisme en général. Le secteur "tourisme" a réalisé directement quelques recherches notamment en 1981 une importante étude sur une mesure économique et sociale, le chèque-vacances. Récemment, le secteur "tourisme" a suscité la création

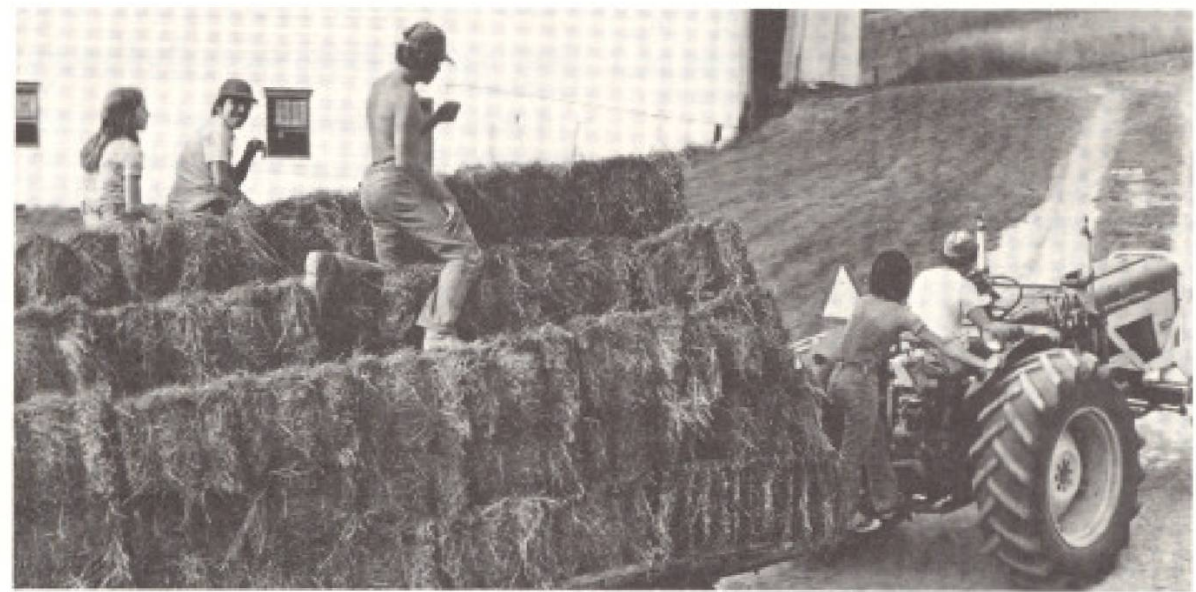

\title{
Les agriculteurs-hôtes et le programme "séjour à la ferme"
}

\begin{abstract}
La Fédération des agricotours du Québec est un organisme sans but lucratif oeuvrant dans le domaine de l'agro-tourisme et du tourisme en milieurural. Une recherche réalisée par Claire Levasseur, Diane Goulet et Lorraine Vaillancourt pour la Fédération des agricotours du Québec consistait à connâ̂tre l'opinion et les motivations des agriculteurs-hôtes offrant le programme "séjour à la ferme".
\end{abstract}

Le "séjour à la ferme" offre la possibilité aux vacanciers de partager le mode de vie des agriculteurs-hôtes en séjournant à la ferme. Les agriculteurs offrent le gitte et la pension complete ainsi que la participation aux activités de la ferme.

Selon l'analyse des données, la motivation première qui pousse un agriculteur à offrir le séjour à la ferme est axée sur le côté social. En effet, la majorité des répondants ont exprimé le désir d'établir de nouveaux contacts et le désir de faire connaître le milieu rural. Par contre, le désir d'avoir un revenu supplémentaire n'a été choisi que dans une proportion de $23.5 \%$.
Le taux de satisfaction le plus élevé face au programme "séjour à la ferme" se retrouve parmi les agriculteurs qui ont comme motivation première d'établir de nouveaux contacts.

Selon les agriculteurs-hôtes, le rapport visiteur-visité semble donner cours à un échange bidirectionnel très fort. Toutefois, on ne semble pas tenir compte de l'avantage pour le visiteur de retrouver une certaine forme d'animation de la part des hôtes puisque cette variable a été ignorée par les répondants dans le choix des éléments les plus caractéristiques.

De cette recherche, il en est ressorti malgré tout que l'aspect économique était comblé puisque la majorité des hôtes trouvaient le programme assez rentable et par le fait même étaient satisfaits d'en faire partie.
De plus, du fait que la connaissance du milieu rural est majoritairement ce qu'ils offrent de mieux aux visiteurs, il s'avère donc qu"un lien s'établit entre le mode de vie urbain et le mode de vie rural.

Un fait intéressant à souligner est que $90 \%$ des répondants sont des femmes. ce qui semble confirmer que l'accueil des vacanciers relève surtout de la responsabilité des femmes.

Les résultats de cette recherche constituent un outil important pour le développement en agro-tourisme et l'amélioration de I"efficacité du programme de la Fédération des agricotours du Québec.

(Resumé par Claire Levasseur) 
du Groupe de ressources en tourisme social du Québec (1415 est, rue Jarry. Montréal H2E 1A7) qui entend être un véritable moteur pour le développement à partir des actions suivantes: études et recherches, soutien technique aux initiatives, programmes d'information et de formation, expérimentation. Les associations elles-même réalisent des études et des recherches lavec l'aide d'étudiants universitaires, de pigistes ou de firmes de consultants). Voici quelques exemples:

Fédération des agricotours du Québec

(1415 est, rue Jarry, Montréal H2E 1A7) Différentes études concernant le développement des programmes, de la clientèle et de la philosophie même de la fédération.

\section{Fédération québécoise de I'ajisme} (1320 ouest, rue Sherbrooke, Montréal H3G 1 H9)

Études et recherches sur le réseau des auberges de jeunesse et les caractéristiques de la clientèle.
Fédération québécoise de camping et de caravaning

(1415 est, rue Jarry, Montréal H2E 1A7) Études sur les relations visiteurs-visités à l'intérieur des programmes de la fédération ("quels sont les facteurs qui incitent les visiteurs à aller voir les visités et ces derniers à recevoir les visiteurs?")

\section{Mouvement québécois des camps familiaux}

(1415 est, rue Jarry, Montréal H2E 1A7) Études au sujet de la clientèle des camps familiaux. Recherches sur les concepts d'aménagement, sur un mobilier adéquat, etc...

\section{Organisation du tourisme étudiant du} Québec

(1415 est, rue Jarry, Montréal H2E 1A7) Recherches sur l'implantation d'un système de coupon d'hébergement ("open voucher") pour les résidences étudiantes du Québec. Étude de I'impact du tourisme équestre en milieu étudiant. EEtudes de marché au plan local pour chacune des institutions.

\section{Kéroul}

(2275 est, Laurier, Montréal H2H 2N8) Études sur les retombées économiques. Études concernant l'accessibilité des lieux pour les personnes handicapées.

Société québéccoise de plein-air (1415 est, rue Jarry, Montréal H2E 1A7) Études portant sur la clientèle des centres et des bases de plein-air.

Enfin, plusieurs asociations touristiques régionales, malgré la jeunesse de leur histoire, se sont lancé dans la recherche pour assurer une meilleure organisation du tourisme dans leur région. C'est par cette voie que I'Association touristique du Bas StLaurent a développé de nouveaux produits afin de mettre en valeur cette région du Québec: observation des baleines et des oies blanches. démonstration du traitement de la laine, randonnées récréatives à pied, en canot, à cheval. L'Association touristique régionale du Coeur du Québec a effectué l'été dernier une évaluation en profondeur du réseau d'accueil et d'information dans sa région dont les conclusions rejoignent celles de la firme Drouin, Paquin et associés.

\title{
Les camps familiaux Profil des vacanciers
}

\begin{abstract}
Résultats d'une enquête portant sur la situation économique et les habitudes de vacances de familles participant aux camps familiaux, par Georges Bertrand, Martin Roy. Bernard Tremblay pour le Groupe ressources-vacances-sites Inc. (maintenant fusionné avec le Mouvement québécois des camps familiaux). Octobre 1980.
\end{abstract}

Les trois objectifs majeurs de cette enquête se résument comme suit: premièrement, dresser un profill de la situation socio-économique et des habitudes de vacances des familles qui participent aux camps familiaux: deuxièmement, établir les caractéristiques comparées pour les nonvacanciers et les usagers des camps familiaux: finalement, identifier et qualifier trois aspects particuliers de l'expérience de vacances vécues par ces mêmes vacanciers. L'étude se déroule selon ces trois divisions, à partir d'un questionnaire passé à tous les chefs de famille (130) présents dans les sites visités par les enquêteurs.

Profil socio-économique: on constate une dichotomie quant aux cellules familiales. Les cellules mono et biparentales possedent des profils distincts non seulement dans leur taille respective mais au niveau du revenu, de l'emploi, du transport, etc. De plus, on dénote un accroissement de la nouvelle clientèle coîncidant avec une nouvelle approche de la promotion du mouvement. On peut s'inquiéter aussi de la sous-représentation des jeunes familles. Enfin, on souhaite que ce profil socio-économique suscite des hypothèses, des observations, des commentaires sur lesquelles s'appuieront d'autres recherches.

Caractéristiques comparées des nonvacanciers et des usagers: en se basant sur cette enquête et d'autres études au sujet des non-vacanciers, on observe une certaine similitude entre la clientèle des camps familiaux et les non-vacanciers. Ainsi, l'accessibilite a des vacances actives pour des familles

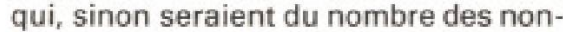
vacanciers, est favorisee par le Mouvement des camps familiaux. On espère, suite à cette étude, des analyses plus complètes afin que le Québec se dote d'un réseau polyvalent d'infrastructures de vacances familiales.

Trois aspects particuliers de ce type de vacances: tout d'abord, les motivations qui ont incité les familles à choisir ce type de vacances et les craintes ressenties à la veille de leur première participation. Ensuite, les facteurs de satisfaction et d"insatisfaction en ce qui concerne le site, l'animation, les services, les équipements de loisirs et tout autre item, selon le cas. Finalement, les aspirations des vacanciers sur l'avenir du Mouvement des camps familiaux.

Ces résultats se veulent à la base d'analyses plus élaborées en vue de continuer cette réflexion déja largement amorcée sur le phénomène des vacances familiales au Québec. 


\section{4) Le milieu universitaire}

Le milieu universitaire est sans contredit un milieu propice à la recherche. Méme si la recherche en tourisme n'est pas trés répandue dans ce milieu, plusieurs études, recherches, enquêtes ont été entreprises ces derniers temps par quelques professeurs bien connus. Cette section ne fera pas état des programmes d'aide à la recherche en milieu universitaire ni des infrastructures susceptibles de soutenir les chercheurs, mais présentera brièvement les travaux de quelques chercheurs universitaires dans le champ du tourisme.

Ainsi, dernièrement Marcel Samson de I'INRS-Urbanisation, en collaboration avec Véronique Barrier, dévoilait les résultats d'une enquête réalisée auprès d'un échantillon de la population de la région métropolitaine de Montréal. Cette étude intitulée Vacances et tourisme 80 a trait aux comportements et aux aspirations des ménages de la région métropolitaine de Montréal en matière de vacances et de tourisme.

Bien entendu I'Universitế du Québec à Montréal fut le lieu de quelques études en tourisme. Ainsi M. Pierre Filiatrault actuellement vice-doyen à la famille des sciences de la gestion, a procédé à une étude commanditée par I'Association des terrains de camping du Québec (A.T.C.Q.) en octobre 80 . Cette étude portait sur le comportement

\section{Sondage auprès de la clientèle des auberges de jeunesse au Québec}

(les résidants au Québec seulement)

Dans le cadre d'une étude sur les auberges de jeunesse du Québec. effectué par Johanne Demers pour la Fédération québécoise de l'ajisme.

a) Au niveau des caractéristiques socio-économiques, l'usager moyen est un homme ou une femme, âgé de 18 à 25 ans, célibataire et habitant Montréal ou Québec. II peut être aussi bien étudiant que travailleur, il possède une scolarité assez élevée et en général, son revenu annuel est inférieur à $\$ 10,000.00$

b) Au niveau des habitudes de voyages, les jeunes itinerants font généralement un seul voyage par été avec utilisation des auberges de jeunesse, voyage qui dure environ une semaine. Le principal concurrent de l'auberge est le camping. Les voyages organisés amènent très peu d'usagers dans les auberges. La clientèle préfère vovager par ses propres movens, sur le pouce, en couple ou avec des amis (surtout)

c) Quant aux modalités d'utilisation des auberges, près de la moitié de la clientèle n'a visité qu'une ou deux auberges au cours de l'été 1979. II apparait que les hommes au contraire des femmes sont les plus grands utilisateurs. Par vovage, on note que les usagers utilisent plus d'auberges 12.5 en moyenne) et a nouveau, les hommes sont les meilleurs consommateurs. De plus, les séjours effectués sont très courts: dans $81 \%$ des cas, il s'étendent sur 2 nuits ou moins. Finalement, certaines régions et certaines auberges attirent les faveurs de la clientèle.

d) Au plan de la satisfaction relative à l'utilisation des auberges, les usagers considerent que parmi les services les plus importants, l'accueil est évalué assez positivement tandis que le score moyen accorde aux services d'hébergement et de resta uration laisse croire quiune amélioration sensible s'impose dans ces deux cas. Les "tarifs" et le "personnel" sont jugés de manière très favorable. Au niveau des services de support ou auxiliaires, les taux de satisfaction vont de moven à faible. Les femmes semblent généralement plus insatisfaites que les hommes, les jeunes le sont plus que les plus âgés, les travailleurs plus que les chómeurs et les étudiants, et qu'enfin les grands utilisateurs d'auberges sont plus satisfaits que les faibles utilisateurs.

e) Au niveau des motifs d'utilisation des auberges, l'étude a fait ressortir trois motivations principales incitant la clientèle à fréquenter les auberges de jeunesse. Ce sont par ordre d'importance: les faibles coûts, la possibilité de rencontrer des gens et l'ambiance.

f) Sur la question des besoins concernant les services ou équipements des auberges, les services de base les plus importants et jugés essentiels par la clientèle sont: I'hébergement, le camping pour les auberges rurales. I'information touristique, la restauration, l'équipement récréatif d'intérieur et la buanderie. Plus des trois-quarts des usagers sont prêts à envisager une hausse de tarif si l'introduction ou l'amélioration de certains services oblige ce changement.

g) Plusieurs opinions furent émises sur certains aspects de la mission, de l'aménagement, du fonctionnement et de l'image des auberges. L'auberge de jeunesse devrait s'adresser a tous les publics peu importe l'áge. Aussi, sa mission fondamentale (hébergement à prix modique) devrait s'accompagner d'un rôle éducatif et de sensibilisation au patrimoine. De plus, la clientèle préférerait les auberges de movenne dimension (41 à 70 lits) et de petite dimension (moins de 41 lits) et également des chambres de plus petite capacite (4 lits et moins). II appert aussi que les usagers souhaiteraient la disponibilité de chambres mixtes, l'instauration d'un couvre-feu vers 23 heures et la permission de consommer de l'alcool à l'auberge. On remarque de plus que les usagers acceptent facilement de participer aux tâches ménagères à l'intérieur des auberges et qu'ils sont satisfaits de la discipline qui y règne. Paradoxalement cependant, ils sont divisés quant à la perception qu"ils ont de l'image que l'auberge de jeunesse projette au niveau du public.

h) Quant à la localisation des auberges, les usagers considèrent que le réseau des auberges de jeunesse couvre assez bien l'ensemble du Québec. Par contre, al l'intérieur des régions la couverture est jugée insatisfaisante par $50 \%$ des répondants. Parmi les régions où, selon les usagers, des auberges devraient être établies, on retrouve celles de Québec, du Bas St-Laurent. Gaspésie, lles de la Madeleine, de Montréal rivenord, du Nord-ouest et de l'Outaouais. La principale raison motivant I'installation d'auberges demeure l'attrait touristique des milieux, mais dans la région en périphérie de Montréal, l'attrait pour le plein-air arrive presque nez-â-nez avec l'attrait touristique. 
des campeurs québécois dans leur choix d'un terrain de camping de I'A.T.C.Q.

Une autre étude remontant en 1977 a permis à $M$. Filiatrault, avec la collaboration de M. J.R. Brent Ritchie. de se pencher sur le processus décisionnel familial dans le choix d'un établissement d'hébergement urbain. L'objectif de cette recherche était d'étudier le processus décisionnel des familles dans le choix d'un hótel/motel. L'étude a été faite auprès de touristes anglophones (Canadiens et Américains) qui résidaient dans des hôtels et motels de la ville et les environs de Québec durant l'été. L'étude a permis d'identifier des différences significatives de comportements entre les familles et les couples et entre les utilisateurs de chaĩnes.

M. Jean Stafford, professeur au module de Gestion et intervention touristiques, a participé à une enquête réalisée pour la Fondation St-Denis. Cette enquête "Sondage St-Denis" visait essentiellement deux objectifs: d'une part dresser le profil de la population qui fréquente le quartier St-Denis et d'autre part mesurer l'intérêt culturel de ces individus.
A l'Université de Sherbrooke, M. Roger Nadeau, professeur au département de géographie, a participé à une étude portant sur l'implantation de casinos au Québec et plus spécifiquement dans la région de I'Estrie. Cette étude visait à faire la démonstration de l'intérêt de l'implantation d'un casino dans la région.

Au cours de l'année 1981, M. Nadeau s'est intéressé aux schémas d'aménagent pour le développement touristique et récréatif du MontMégantic. Réalisé à la demande du M.I.C.T., cette étude visait le développement touristique de cette région.

\title{
Étude de comportement des campeurs québécois
}

\author{
Une étude réalisée par Pierre Filiatrault pour le compte de l'Association des \\ terrains de camping du Québec, Laboratoire de recherches en sciences \\ administratives, UOAM, octobre 1980.
}

Les informations recueillies devraient faciliter la prise de décision des membres du conseil d'administration de cette association en vue d'une amélioration des services offerts aux campeurs itinérants et saisonniers. Parmi les objectifs secondaires, on retrouve une meilleure connaissance des habitudes de camping des campeurs, l'identification des critères importants dans le choix d'un terrain de camping. I'évaluation de ces terrains, etc...

A travers cette enquête, on découvre que le site de camping sert de lieu de résidence ou de vacances à la majorité des campeurs, ceux-ci séjournant sur le même terrain tout l'été ou toute l'année. La décision de prendre des vacances et même celle du choix de leur terrain de camping furent prises l'été précédent, pour la majorité des répondants. Ceci n'est pas étonnant, sachant que près de $80 \%$ des. répondants font du camping saisonnier.

Le choix d'un terrain de camping est basé sur certains critères dont le plus important est la propreté, suivi de l'attitude "écologique" des autres campeurs, de la disponibilité des services de base, du bon ordre (contrôle, règlements), des activités et loisirs organisés, de l'accessibilité à la baignade. L'importance accordée à la proximité des sites de pèche, d'un terrain de golf et des centres de sports d'hiver est moindre. Enfin les activités mondaines n'ont pas la faveur des répondants.
L'évaluation du terrain de camping. faite par les répondants, par rapport à certains criteres fut assez positive. surtout en ce qui concerne les services. Par contre, l'évaluation de l'entretien, des douches et des toilettes ainsi que celles des loisirs, des activités organisées et des terrains de jeux sont plutôt faibles alors que l'importance accordée à ces mêmes services est relativement élevée.
On relève de plus que la connaissance de I'A.T.C.O. auprès des campeurs est plutôt faible. Par contre le "guide du camping" publié par I'A.T.C.Q. est davantage connu et utilisé. On y préfère dans l'ordre, la liste des terrains et services, l'information générale sur le camping et la carte routière qui localise les terrains. Toutefois, on apprécierait davantage ce guide s'il était disponible dès l'hiver.

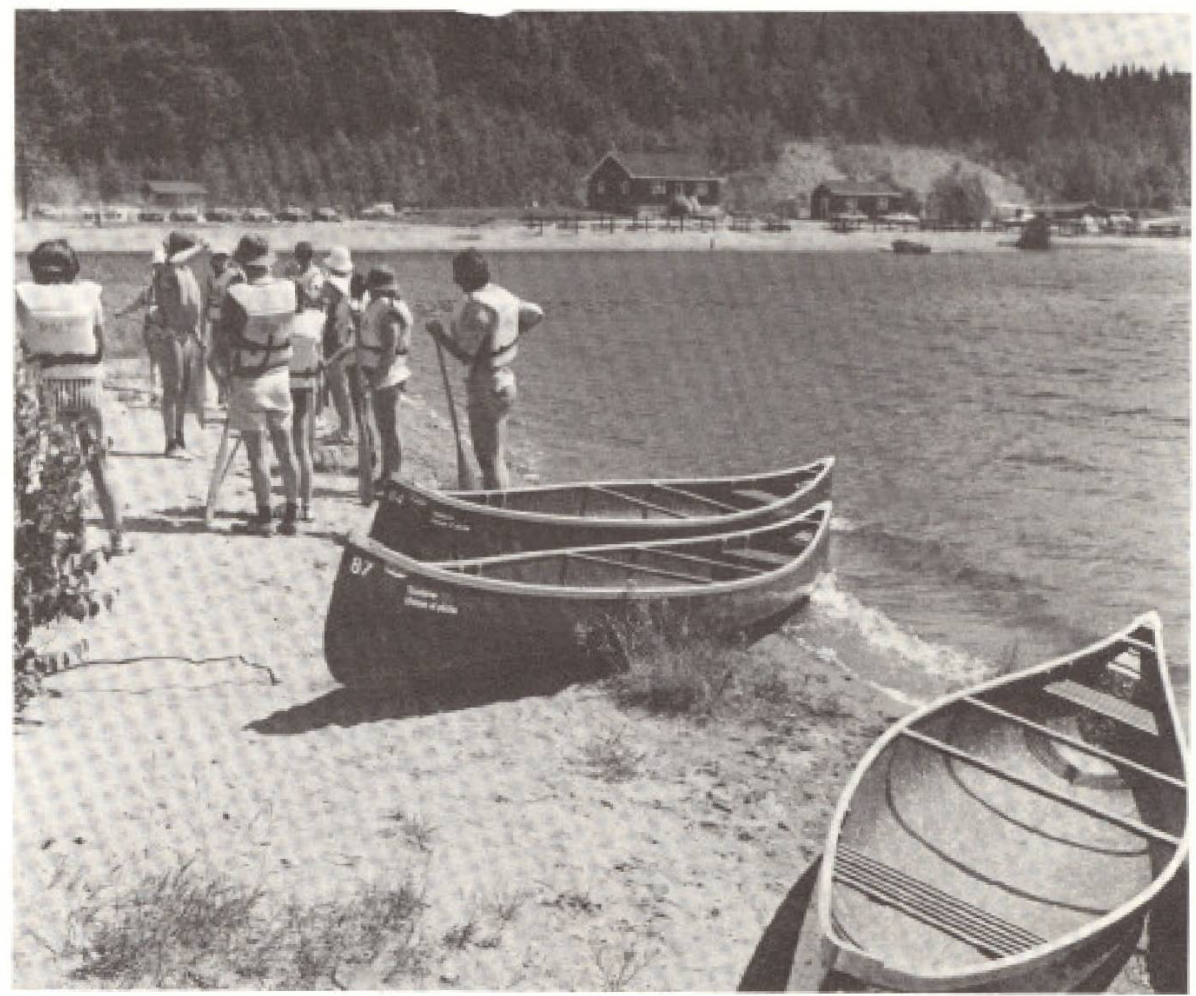


L'été 82 permettra à $M$. Nadeau de participer à un camp de travail portant sur un projet de recherche axé sur la clientèle québécoise qui va vers la Nouvelle-Angleterre pour les vacances. Une distribution de questionnaires, pour fins de statistiques sur l'utilisation du sol et les motivations des Québécois, favorisera une réflexion sur d'éventuelles possibilités de développement de régions au Québec dans le but de répondre aux motivations des Québécois.
Finalement, $M$. Nadeau débute au mois de juin 82 un projet de recherche sur le tourisme social en collaboration avec M. Marc Laplante, directeur du module de Gestion et intervention touristiques de I'UQAM. Cette recherche permettra de déterminer, entre autre, les causes et les besoins des non-partants.
En terminant, il serait pertinent de mentionner que $M$. André Barabé, professeur à I'Université du Québec à Trois-Rivières, procède présentement à une étude sur l"impact psychosociologique des usagers des équipements récréatifs dans les espacies naturels protégés. Cette étude servira à déterminer le seuil optimum de tolérance de ces équipements ainsi qué la réaction des gens face à l"isolement et à la solitude.

\title{
Vacances et tourisme 80
}

\begin{abstract}
Une enquête auprès de la population de la région métropolitaine de Montréal, par Marcel Samson et Véronique Barnier. INRS-Urbanisation. collection "Études et documents" no 30, mai 1982. 149 p. et 5 cartes."
\end{abstract}

a) Les non-vacanciers $-28.9 \%$ de la population montréalaise n'a pas pris de vacances comparativement à $25 \%$ en 1970 - $21 \%$ des répondants n'ont pas pris de vacances depuis les trois dernières années comparativement â $16 \%$ en 1970 . Le pourcentage des nonvacanciers est plus élevé chez les allophones que chez les francophones ou les anglophones. De plus, ils appartiennent aux catégories de revenu les plus faibles et se recrutent parmi les personnes qui ne font pas partie de la force active de travail. La proportion augmente dans les tranches d'äge "les plus jeunes" et "les plus âgées". Les raisons principales invoquées pour les non-vacances sont d'ordre économique $(44.3 \%)$, les obligations familiales $(32.5 \%)$, les contraintes occupationnel= les $(23.2 \%)$.

b) Les vacanciers - En 1980 ils représentent $71.1 \%$ ds individus alors qu'ils étaient $75 \%$ en 1970 . Par contre les vacanciers non-partants de 1980 comptent pour $9.9 \%$ des répondants (en $1970,15 \%)$ et les vacanciers partants pour $61.2 \%$ (en $197060 \%$ ). Ainsi on peut dire que les non-partants représentent $14 \%$ de l'ensemble des vacanciers et que les partants à destination unique forment $61.8 \%$ de ce mème contingent et les partants à destinations multiples, $24.2 \%$.

c) Période des vacances - Surtout au mois de juillet et aoüt ( $53.8 \%$ ). Toutefois $24.4 \%$ d'entre eux choisissent les mois d'hiver (décembre à fin mars). En 1970 ils étaient $86 \%$ à prendre leurs vacances entre le 1 er avril et le 30 septembre et $14 \%$ entre le 1 er octobre et le 31 mars. d) Durée des vacances - Pour une semaine de vacances, la proportion en 1980 est la même qu'en 1970 soit $10.7 \%$. Pour 2 semaines, ils ne sont plus que $30.1 \%$ en 1980 comparativement à $42 \%$ en 1970. Par contre plus de gens ont des vacances de 3 semaines ou plus, soit $59.3 \%$ en 1980 comparativement à $46 \%$ en 1970 .

e) Destination des vacanciers $50.3 \%$ des partants à destination unique choisissent le Québec surtout dans les régions voisines de Montréal avec une forte concentration dans les Laurentides. Les partants à destinations multiples $(34.2 \%)$ se rendent majorité à l'extérieur de la province $(64,4 \%)$. Pour ceux qui demeurent au Québec, ils préfèrent les zones plus lointaines comme la Gaspésie. Les États-Unis constituent la destination privilégiée de la totalité des partants, soit pour $33 \%$, et la Floride demeure l'endroit favori. II est intéressant de remarquer que $86.1 \%$ des partants à destination unique, anglophones $(75 \%$ en 1970) choisissent l'extérieur du Québec. $34.8 \%$ font de même chez les francophones $(33 \%$ en 1970$)$. Finalement, plus les Montréalais ont une scolarité ou des revenus élevés, plus ils partent à l'extérieur de la province.

f) L'hébergement des vacanciers Pour les partants à destination unique, c'est la maison d'amis ou de parents $(32.8 \%)$ et le chalet $(18.8 \%)$. Pour les partants à destinations multiples, ils se répartissent également entre le camping, I'hồtel et le motel.

g) Autres éléments de l'organisation des vacances - Lautomobile est de loin le moyen de transport le plus utilisé. Excepté I'avion, les autres moyens sont délaissés. h) Comportements touristiques de week-end - Le tiers de la population (34.1\%) est sorti fréquemment de la région montréalaise à des fins de loisirs et ce en dehors des vacances annuelles. $38.6 \%$ de la population se serait déplacée quelques fois durant l'année. Plus de vacanciers $(38.6 \%)$ que de nonvacanciers $(23.3 \%)$ sortent fréquemment de la région métropolitaine en dehors de la période de vacances. En été, les régions préférées sont les Laurentides $(26.7 \%)$, I'Estrie $(10.6 \%)$ et les États-Unis (10.6\%). L'hiver, les Laurentides attirent $44.1 \%$ de la population pour $11.5 \%$ en Estrie et $5.6 \%$ pour les États-Unis.

i) Aspirations et projets de vacances - Au moment de l'enquête (fin mai 1981) seulement $42 \%$ des individus avaient des projets de vacances pour 1981. Bien entendu les vacanciers de 1980 font plus de projets $(50.6 \%)$ que les gens qui n'ont pas eu de vacances $(22.5 \%)$.

j) Excursions à l'intérieur de la région métropolitaine - Seulement $37.5 \%$ des montréalais ont fait des excursions à l'intérieur de la région de Montréal Une personne sur 2 a utilisé au moins une fois certains equipements à rayonnement régional comme le stade olympique etc...

Cette enquéte met à jour des données recueillies par Marcel Samson et ses collaborateurs pour Tannee 1970 Montpetit, M. Samson, M. et Lamonde P. Vacances ot tourisme. Presses de l'Université du Ouébec à Montréal. 1972 . 